\title{
Intrinsic spin-relaxation induced negative tunnel magnetoresistance in a single-molecule magnet
}

\author{
Haiqing Xie, ${ }^{1}$ Qiang Wang, ${ }^{1}$ Hai-Bin Xue, ${ }^{2}$ HuJun Jiao, ${ }^{1}$ and J.-Q. Liang ${ }^{1, \text { 田 }}$ \\ ${ }^{1}$ Institute of Theoretical Physics and Department of Physics, Shanxi University, Taiyuan 030006, China \\ ${ }^{2}$ College of Physics and Optoelectrics, Taiyuan University of Technology, Taiyuan 030024, China
}

(Dated: November 8, 2018)

\begin{abstract}
We investigate theoretically the effects of intrinsic spin-relaxation on the spin-dependent transport through a single-molecule magnet (SMM), which is weakly coupled to ferromagnetic leads. The tunnel magnetoresistance (TMR) is obtained by means of the rate-equation approach including not only the sequential but also the cotunneling processes. It is shown that the TMR is strongly suppressed by the fast spin-relaxation in the sequential region and can vary from a large positive to slight negative value in the cotunneling region. Moreover, with an external magnetic field along the easy-axis of SMM, a large negative TMR is found when the relaxation strength increases. Finally, in the high bias voltage limit the TMR for the negative bias is slightly larger than its characteristic value of the sequential region, however it can become negative for the positive bias caused by the fast spin-relaxation.
\end{abstract}

PACS numbers: 75.50.Xx, 75.76.+j, 85.65.+h, 85.75.d

\section{INTRODUCTION}

The electron transport through magnetic molecules has attracted much attention recently in molecular spintronics $\underline{\underline{1-27}}$, because of its potential applications in information storage and processing. The single-molecule magnet (SMM), which has a high intrinsic spin and an easy-axis magnetic anisotropy, is of particular interest. Some peculiar phenomena such as Coulomb-blockade, magnetic excitations, complete current-suppression, and negative differential conductance have been observed experimentally in the quantum transport through a $\mathrm{SMM}^{4}$. The Berry phase $e^{\frac{5}{}}$ and Kondo effects ${ }^{6}$ on the average current (or differential conductance) are predicted theoretically. Moreover, the full counting statistics (current fluctuation $)^{7}$ and spin fluctuation of magnetic molecules ${ }^{8}$ are also studied. On the other hand, the magnetic switching of SMM is realized by thermal spin-transfer torque ${ }^{9}$, spin-bias $\frac{10}{}$, and spin-polarized current injected from ferromagnetic $(\mathrm{FM})$ electrodes ${ }^{8,11}$-14. It is predicted that the spin-current polarization can be reversed through a SMM with FM leads $\frac{15}{}$ and even a pure spincurrent can be generated in normal leads by the thermoelectric effects 16 . Spin-diode behavior ${ }^{17}$ and memristive properties $\underline{18}$ are also observed in spin-polarized transport through a SMM. The current difference between the parallel (P) and antiparallel (AP) configurations of two FM electrodes known as the tunnel magnetoresistance $(\mathrm{TMR})^{28}$ was systematically investigated for the SMM by Misiorny et al. in the sequential, cotunneling, and Kondo regions $19-21$. The TMR is positive usually, since the $\mathrm{P}$ current is greater than the AP one, while a large negative TMR is shown to exist in the SMM ${ }^{19}$. Furthermore, the tunneling anisotropic magnetoresistance (TAMR) with tunable magnitude and sign is found in SMM junctions with only one FM electrode $\underline{22}$.

A long spin-relaxation time of the SMM is required in the quantum information processing 29 and information storage $\frac{13}{2}$. However, in addition to the spin-relaxation induced by the electron tunneling through molecules, the SMM itself may have intrinsic spin-relaxation ${ }^{12.23}$, which originates from the interactions with environmental spins. A short spin-relaxation time is found in a recent experiment ${ }^{24}$ in which a spin-polarized scanning tunneling microscopy is used to pump the spins of Mn atoms. Moreover, the experimentally observed quenching of conductance steps 25 has been successfully explained in terms of the spin-relaxation 26 . The effects of intrinsic spinrelaxation on the current-induced magnetic switching of SMM have been studied theoretically $\underline{12}$. When the relaxation of SMM is very fast, the fine structure of transport is suppressed, but a high spin-polarized current in nonmagnetic leads can be generated with an external magnetic field 23 . For the quantum-dot (QD) systems, on the other hand, the intrinsic spin-relaxation can result in the TMR suppression ${ }^{30}$ and reversal ${ }^{31}$ in the sequential and cotunneling regions, respectively. It is also shown that the peak heights of differential conductance in QDs are increased by the spin-relaxation ${ }^{32}$.

In this paper we study the spin-polarized transport through a SMM with FM leads in both sequential and cotunneling regions and pay particular attention on the intrinsic spin-relaxation, which affects remarkably the transport properties. A large negative TMR can be obtained when a magnetic field is applied along the easyaxis of SMM. Sec. II is devoted to the rate-equation formalism for the spin-polarized transport through a SMM and the related TMR. We demonstrate and discuss the effects of intrinsic spin-relaxation on the current, differential conductance, magnetization and TMR based on the numerical results in Sec.III. Finally, the conclusion is given in Sec. IV. 


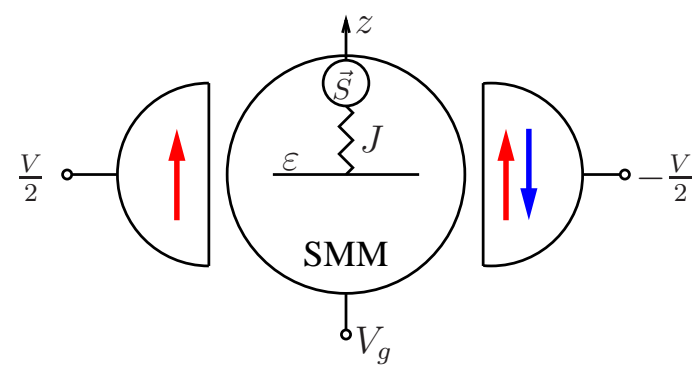

FIG. 1: (Color online) Schematic diagram of electron transport through a SMM weakly coupled to ferromagnetic electrodes. The magnetizations of the two leads are collinear with the magnetic easy axis of SMM (as $\mathrm{z}$ axis). The left (L) and right $(\mathrm{R})$ electrodes are connected with the bias voltage $\mathrm{V} / 2$ and $-\mathrm{V} / 2$, respectively.

\section{MODEL AND METHOD}

The schematic diagram of electron transport through a SMM coupled to two external FM leads is shown in Fig. 1. It is assumed that the lead-magnetizations are collinear with the magnetic easy-axis of SMM in either $\mathrm{P}$ or AP configuration. The total Hamiltonian of the system can be written as $13,19,23,27$

$$
H=H_{\text {leads }}+H_{S M M}+H_{T} .
$$

The first term $H_{\text {leads }}=\sum_{\alpha=\mathbf{L}, \mathbf{R}} \sum_{\mathbf{k} \sigma} \varepsilon_{\alpha \mathbf{k}} c_{\alpha \mathbf{k} \sigma}^{\dagger} c_{\alpha \mathbf{k} \sigma}$ describes noninteracting electrons in electrodes, where $c_{\alpha \mathbf{k} \sigma}^{\dagger}$ $\left(c_{\alpha \mathbf{k} \sigma}\right)$ is the creation (annihilation) operator for an electron with wave vector $\mathbf{k}$ and spin $\sigma$ in the lead $\alpha$, and $\varepsilon_{\alpha \mathbf{k} \sigma}$ is the corresponding electron energy. The spin polarization of FM lead $\alpha$ is defined as $p_{\alpha}=\left(\rho_{\alpha+}-\right.$ $\left.\rho_{\alpha-}\right) /\left(\rho_{\alpha+}+\rho_{\alpha-}\right)$, with $\rho_{\alpha+(-)}$ denoting the density of states for the majority (minority) electrons. The second term

$$
\begin{aligned}
H_{S M M} & =\sum_{\sigma}\left(\varepsilon-e V_{g}\right) d_{\sigma}^{\dagger} d_{\sigma}+U d_{\uparrow}^{\dagger} d_{\uparrow} d_{\downarrow}^{\dagger} d_{\downarrow}-J \mathbf{s} \cdot \mathbf{S} \\
& -K\left(S^{z}\right)^{2}-B\left(s^{z}+S^{z}\right)
\end{aligned}
$$

denotes the SMM Hamiltonian, where the operator $d_{\sigma}^{\dagger}\left(d_{\sigma}\right)$ creates (annihilates) an electron in the lowest unoccupied molecular orbital (LUMO) level and $\mathbf{s} \equiv$ $\sum_{\sigma \sigma^{\prime}} d_{\sigma}^{\dagger}\left(\sigma_{\sigma \sigma^{\prime}} / 2\right) d_{\sigma^{\prime}}$ is the electron spin operator with $\sigma$ being the vector of Pauli matrices. The single-electron energy $\varepsilon$ in the LUMO level is tunable by gate voltage $V_{g}$. The Coulomb energy denoted by $U$ is for the double occupancy of the LUMO level. The exchange coupling $J$ between the local spin of molecule $\mathbf{S}$ and the LUMO electron-spin s can be either FM $(J>0)$ or antiferromagnetic $(\mathrm{AFM})(J<0)$. The easy-axis anisotropy of the SMM is characterized by the parameter $K(K>0)$ and an external magnetic field $B$ is applied along the easyaxis, where $B$ contains the factor $g \mu_{B}$. Since the SMM Hamiltonian $H_{S M M}$ commutes with $z$ component $S_{t}^{z}$ of the total spin operator $\mathbf{S}_{t} \equiv \mathbf{s}+\mathbf{S}$, it can be easily diagonalized in terms of molecular many-body eigenstates $\left|n, S_{t} ; m\right\rangle$ with $n$ denoting the charge state, $S_{t}$ the total spin quantum-number, and $m$ the eigenvalues of $S_{t}^{z}$.

The tunneling Hamiltonian between the LUMO level and leads is $13,19,23,27$

$$
H_{T}=\sum_{\alpha \mathbf{k} \sigma}\left(t_{\alpha} c_{\alpha \mathbf{k} \sigma}^{\dagger} d_{\sigma}+t_{\alpha}^{*} d_{\sigma}^{\dagger} c_{\alpha \mathbf{k} \sigma}\right),
$$

where the parameter $t_{\alpha}$ is the tunneling couplingconstant between the molecule and lead $\alpha$, and the corresponding spin-dependent tunnel-coupling strength is denoted by $\Gamma_{\alpha \sigma}=2 \pi \rho_{\alpha \sigma}\left|t_{\alpha}\right|^{2}$. Furthermore, in terms of the spin polarization $p_{\alpha}$ of lead $\alpha$, we can rewrite the tunnel-coupling strength as $\Gamma_{\alpha \pm}=\Gamma_{\alpha}\left(1 \pm p_{\alpha}\right) / 2$ for the spin-majority (spin-minority) electrons of the lead $\alpha$, with $\Gamma_{\alpha}=\Gamma_{\alpha+}+\Gamma_{\alpha-}$.

Since the SMM-electrode coupling is assumed to be sufficiently weak, i.e., $\Gamma_{\alpha \sigma} \ll k_{B} T$, the molecule relaxes rapidly to the eigenstates of $H_{S M M}$ (rapid dephasing) 23 . Therefore, the rate-equation approach can be adopted to study spin-dependent transport through the SMM. Here, we consider phenomenologically the intrinsic spinrelaxation processes, which may drive the SMM to an eigenstate of lower energy. The transport dynamics is well described by the following rate equation,

$$
\begin{aligned}
\frac{d P_{i}}{d t} & =\sum_{\alpha \alpha^{\prime} i^{\prime} \neq i}\left[-\left(W_{\alpha}^{i, i^{\prime}}+W_{\alpha, \alpha^{\prime}}^{i, i^{\prime}}+W_{r e l}^{i, i^{\prime}}\right) P_{i}\right. \\
& \left.+\left(W_{\alpha}^{i^{\prime}, i}+W_{\alpha^{\prime}, \alpha}^{i^{\prime}, i}+W_{r e l}^{i^{\prime}, i}\right) P_{i^{\prime}}\right],
\end{aligned}
$$

with $P_{i}$ denoting the population probability of the SMM state $|i\rangle$. The sequential rate $W_{\alpha}^{i, i^{\prime}}$ describes the transition from the molecule state $|i\rangle$ to $\left|i^{\prime}\right\rangle$ along with the electron tunneling into or out of lead $\alpha$, and $W_{\alpha, \alpha^{\prime}}^{i, i^{\prime}}$ denotes the cotunneling rate from lead $\alpha$ to $\alpha^{\prime}$. The above sequential and cotunneling rates can be calculated in terms of the $T$-matrix with the help of generalized Fermi goldenrule $15,33-37$. Moreover, the transition selection-rules of spin relaxation are $\Delta n=0$ and $\Delta m= \pm 1$, which are similar to the cotunneling processes. The phenomenological spin-relaxation rate from the state $|i\rangle$ to $\left|i^{\prime}\right\rangle$ reads ${ }^{12,23}$

$$
W_{r e l}^{i, i^{\prime}}=\frac{1}{\tau_{r e l}} \frac{1}{1+e^{\left(\varepsilon_{i^{\prime}}-\varepsilon_{i}\right) / k_{B} T}},
$$

where $\tau_{r e l}$ is the relaxation time and $\varepsilon_{i}$ denotes the energy of state $|i\rangle$. In addition, a dimensionless parameter $\gamma=\hbar /\left(\Gamma \tau_{r e l}\right)$ called the spin-relaxation strength is introduced to compare $\tau_{r e l}$ with the sequential tunneling time $\hbar / \Gamma$.

For the steady-state transport we have the stationary probabilities with the condition $\frac{d P_{i}}{d t}=0$. Therefore, the stationary current through the lead $\alpha$ is given by

$$
\begin{aligned}
I_{\alpha} & =(-1)^{\delta_{\alpha L}} e \sum_{\alpha^{\prime} \neq \alpha, i i^{\prime}}\left[\left(n_{i^{\prime}}-n_{i}\right) W_{\alpha}^{i, i^{\prime}} P_{i}\right. \\
& \left.+\left(W_{\alpha, \alpha^{\prime}}^{i, i^{\prime}}-W_{\alpha^{\prime}, \alpha}^{i, i^{\prime}}\right) P_{i}\right],
\end{aligned}
$$


where the current flows from the left electrode to right one, and the magnetization of SMM is $\left\langle S_{t}^{z}\right\rangle=\sum_{i} m_{i} P_{i}$. The TMR is defined as

$$
\mathrm{TMR}=\frac{I_{P}-I_{A P}}{I_{A P}},
$$

where $I_{P}\left(I_{A P}\right)$ denotes the current through the SMM in the $\mathrm{P}(\mathrm{AP})$ configuration.

\section{RESULTS AND DISCUSSION}

We now analyze the effects of intrinsic spin-relaxation in both the sequential and cotunneling regions with the assumption that the bias voltage is symmetric at the SMM-electrode tunnel junction, i.e., $\mu_{L}=-\mu_{R}=V / 2$. In the following numerical calculations, the parameters are chosen as $S=2, \varepsilon=0.5 \mathrm{meV},|J|=0.2 \mathrm{meV}$, $U=1 \mathrm{meV}, K=0.05 \mathrm{meV}, \Gamma=\Gamma_{L}=\Gamma_{R}=0.001$ $\mathrm{meV}, p_{L}=p_{R}=0.5$ and $k_{B} T=0.04 \mathrm{meV}$.

The TMR value indicated by color index as a function of the gate $\left(V_{g}\right)$ and bias $(V)$ voltages is plotted in Fig. 2. The electron transports are dominated by cotunneling transitions in the voltage-regions labeled with the numbers $n=0,1,2$. While in the rest of regions the transports are dominated by sequential processes. The left panels [(a)-(c)] of Fig. 2 are referred to the results without the intrinsic spin-relaxation as a comparison, and the corresponding TMR for the relaxation strength $\gamma=1$ is shown on the right panels [(d)-(f)]. Comparing Figs. 2(a)-2(c) and Figs. 2(d)-2(f), we can see the obvious variation of TMR induced by the intrinsic spin-relaxation, which in most cases leads to the TMR suppression. However, when an external magnetic field is applied along the easy-axis of SMM, the TMR can be increased seen from the Figs. 2(c) and (f). In particular, a negative TMR value extends from cotunneling to sequential regions.

The bias voltage dependence of current, differential conductance, magnetization of SMM, and TMR are shown in Fig. 3 for different relaxation strength $\gamma$ with the gate voltage $V_{g}=-0.3 \mathrm{mV}$, at which the ground state of SMM is $|0,2 ; \pm 2\rangle$. For the $\mathrm{P}$ configuration of FM leads, the conductance peak-1 [Fig. $3(\mathrm{~b})]$, which corresponds to the transition $|0,2 ; \pm 2\rangle \Leftrightarrow$ $|1,5 / 2 ; \pm 5 / 2\rangle$, appears with the increase of bias voltage $V$ and is not affected by the intrinsic spin-relaxation since the transition is between the ground states of $n=0$ and $n=1$. When the initial state is excited, the corresponding peak- $2(|0,2 ; \pm 1\rangle \Leftrightarrow|1,3 / 2 ; \pm 3 / 2\rangle)$ and peak-4 $(|1,3 / 2 ; \pm 3 / 2\rangle \Leftrightarrow|2,2 ; \pm 2\rangle)$ disappear ${ }^{23}$ in the existence of spin-relaxation. However, the peak-3 $(|0,2 ; \pm 2\rangle \Leftrightarrow|1,3 / 2 ; \pm 3 / 2\rangle)$ still exists and especially the height of peak-5 $(|1,5 / 2 ; \pm 5 / 2\rangle \Leftrightarrow|2,2 ; \pm 2\rangle)$ increases along with the population probability of the ground state $|1,5 / 2 ; \pm 5 / 2\rangle$. This enhancement phenomenon results from the relaxation induced decay of excited states. In consequence, the current $I_{P}$ between the peak-1 and
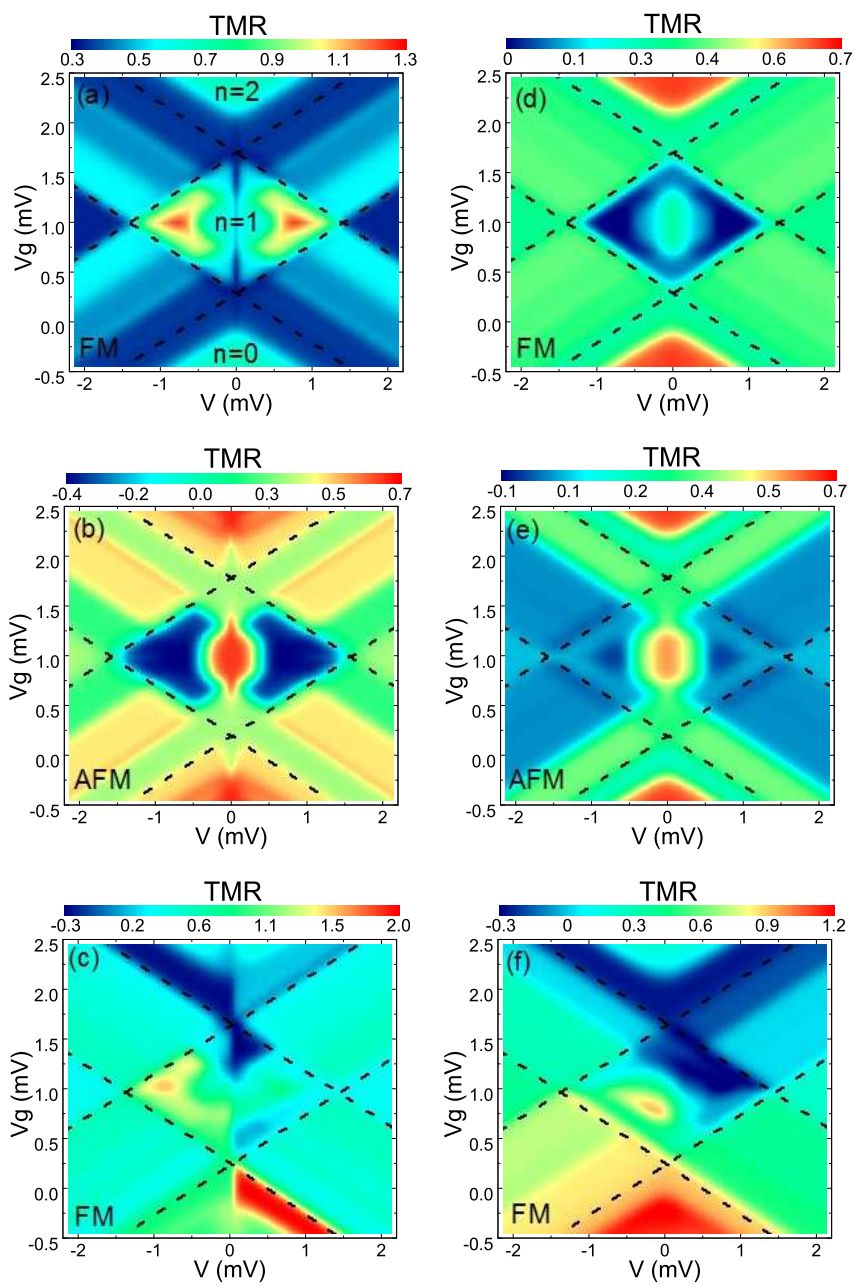

FIG. 2: (Color online) TMR as a function of the bias and gate voltages in the case of FM coupling [(a),(d)], AFM coupling $[(\mathrm{b}),(\mathrm{e})]$, and FM coupling with a magnetic field along the easy-axis of SMM [(c),(f)] for different relaxation strength $\gamma=$ 0 (left panel) and $\gamma=1$ (right panel). The parameters are: $S=2, \varepsilon=0.5 \mathrm{meV},|J|=0.2 \mathrm{meV}, U=1 \mathrm{meV}, K=0.05$ $\mathrm{meV}, k_{B} T=0.04 \mathrm{meV}, p_{L}=p_{R}=0.5, \Gamma=\Gamma_{L}=\Gamma_{R}=0.001$ $\mathrm{meV}$, and $I_{0}=2 e \Gamma / \hbar$.

peak-3 is slightly increased, while the current between the peak- 3 and peak- 5 is decreased as shown in the Fig. $3(\mathrm{a})$. When the bias voltage is high enough such that all channels enter into the transport window, the current $I_{P}$ remains in a constant value independent of the relaxation. In addition, the magnetization of SMM vanishes when the relaxation strength increases to $\gamma=10^{3}$ as shown in Fig. 3(c) (dotted line). The transport properties of AP configuration are quite different from the $\mathrm{P}$ case. For example, in the absence of spin-relaxation the magnetization of SMM [Fig. 3(g)] tends to a large negative value resulting from the electron spin-flip process $\frac{19}{}$, and the steady transport is dominated by the negativeeigenvalue states of $S_{t}^{z}$. The spin-relaxation, however, removes the above tendency and more positive-eigenvalue states contribute to the transport. Therefore, the current 

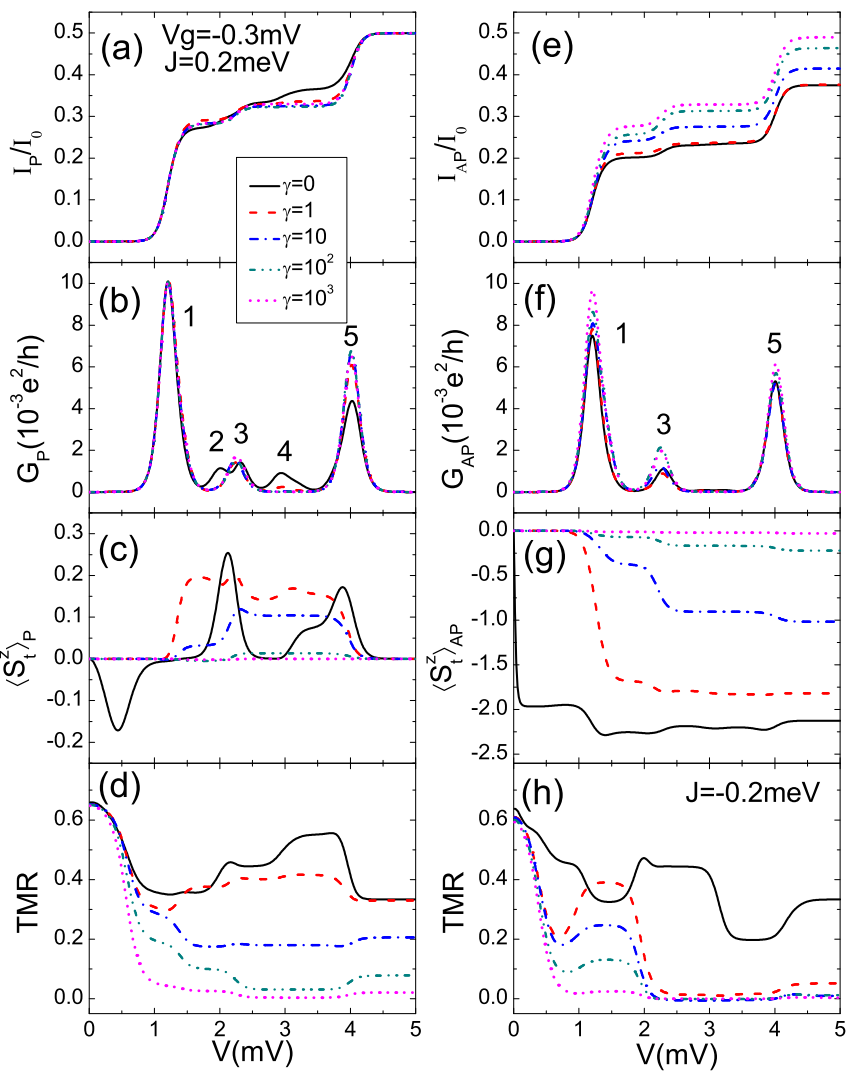

FIG. 3: (Color online) The bias voltage dependence of current $I$, differential conductance $G$, and the magnetization of the SMM for the parallel [left panel (a)-(c)] and antiparallel [right panel (e)-(g)] configurations with the gate voltage $V_{g}=-0.3$ $\mathrm{mV}$. TMR plots are shown in (d) and (h) for the FM and AFM exchange couplings, respectively.

$I_{A P}\left[\right.$ Fig. 3(e)] and differential conductance $G_{A P}$ [ Fig. $3(\mathrm{f})]$ increase monotonously with the relaxation strength $\gamma$. The $\gamma$-dependence of TMR spectrum for both cases of FM and AFM exchange couplings are shown in Fig.3 (d) and (h), respectively. At the low bias voltage, electron transport is dominated by elastic cotunneling processes via ground states of $n=0$ and the TMR is essentially not affected by the relaxation. With the increase of bias voltage, the inelastic cotunneling plays a role in the transport and the current $I_{A P}$ increases due to the intrinsic spinrelaxation. Therefore, the TMR almost vanishes at the large value of $\gamma$. In the sequential tunneling region, the fast relaxation also leads to vanishing TMR. In addition, the energy of state $|1,3 / 2 ; m\rangle$ for the AFM exchange coupling is lower than that of the state $|1,5 / 2 ; m\rangle$, and the TMR decreases (with the increase of relaxation strength) faster compared with the FM case.

In Figs. 4(a)-4(c), we display the current, differential conductance and magnetization of SMM in the P configuration as a function of the bias voltage $V$ for the FM exchange coupling and $V_{g}=1 \mathrm{mV}$. In this case, the gate voltage corresponds to the electron-hole symmetry point, thus the magnetization of SMM is always zero indepen-
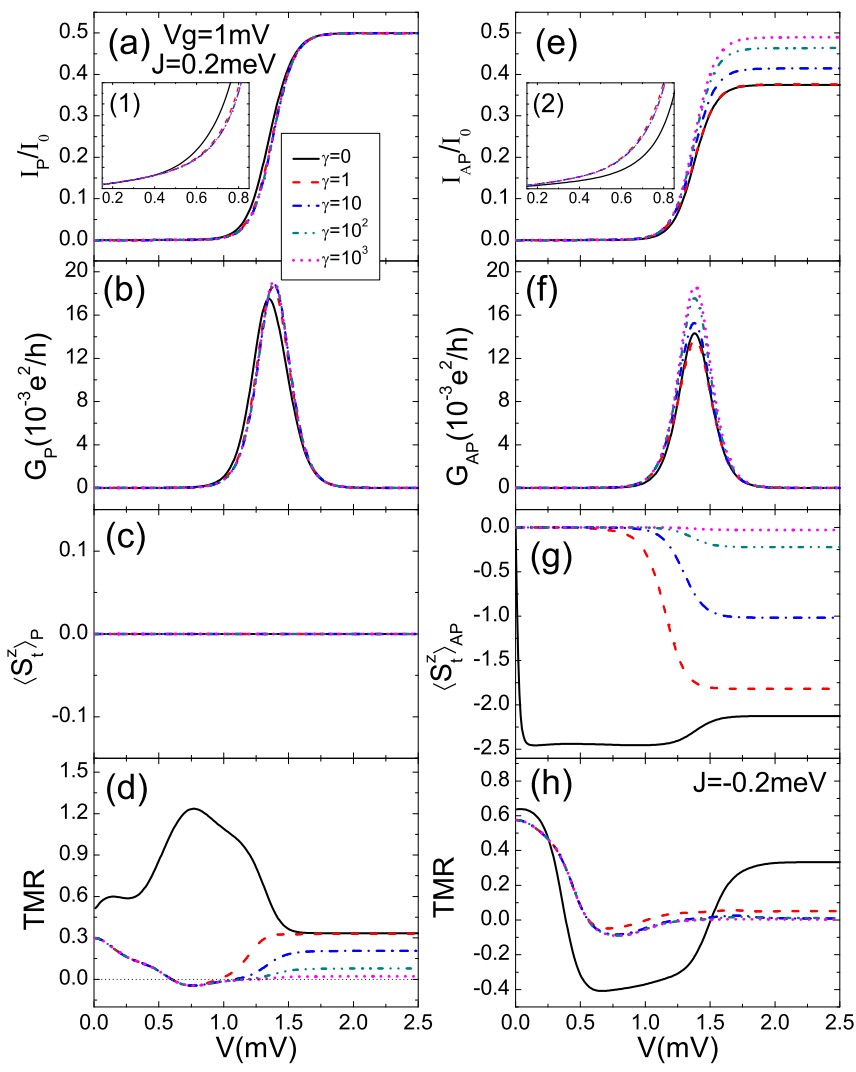

FIG. 4: (Color online) The bias voltage dependence of $I_{P}$ (a), $I_{A P}(\mathrm{e}), G_{P}$ (b), $G_{A P}$ (f), $\left\langle S_{t}^{z}\right\rangle_{P}$ (c) $\left\langle S_{t}^{z}\right\rangle_{A P}(\mathrm{~g})$, and TMR for the FM [(d)], AFM [(h)] exchange couplings with the gate voltage $V_{g}=1 \mathrm{mV}$. The cotunneling currents $I_{P}$ and $I_{A P}$ are shown in the insets of (a) and (e), respectively.

dent of the bias voltage $V$ [Fig. 4(c)] and the spectrum of differential conductance has only one peak seen from Fig. 4(b). Moreover, the peak-position has a slight shift in the existence of intrinsic spin-relaxation due to the increase of population probability of state $|1,5 / 2 ; \pm 5 / 2\rangle$. For the $\mathrm{P}$ configuration, it can be seen from Fig. 4(a) that $I_{P}$ is suppressed by the spin-relaxation in both the cotunneling [the inset of Fig. 4(a)] and sequential tunneling regions. But for the AP configuration, the current [Fig. 4(e)] and differential conductance [Fig. 4(f)] are enhanced by the relaxation. In the Fig. 4 (d), we show the bias voltage dependence of TMR with FM exchange coupling for different relaxation strengths. The intrinsic spin-relaxation leads to the decrease of TMR from a large positive to small negative value in the cotunneling region. This can be understood from the behaviors of $I_{P}$ and $I_{A P}$ [see the insets of Figs. 4(a) and 4(e)]. For the AFM exchange coupling, the TMR exhibits a different behavior from the FM case. At low bias voltage, in which electron transport is dominated by cotunneling processes via ground states $|1,3 / 2 ; \pm 3 / 2\rangle$, the intrinsic spin-relaxation has a little effect on the TMR. When the bias voltage increases, the enhancement of TMR induced by the spinrelaxation becomes obvious. However, the large negative 
TMR almost disappears due to the fast relaxation [Fig. 4(h)]. The above behavior of TMR for the AFM coupling is due to the decrease of $I_{A P}$ in the cotunneling region. When all of transport channels are open in the sequential region, the TMR of both FM and AFM exchange couplings approaches zero when the relaxation strength increases to a sufficiently large value.

Figure 5 shows the effects of the intrinsic spinrelaxation on the bias voltage dependence of TMR in the existence of a magnetic field, which is applied along the easy-axis of SMM. The longitudinal magnetic field lifts the degeneracy of states $\left|n, S_{t} ; \pm m\right\rangle$ and the fast spinrelaxation leads to the transition from the state $\left|n, S_{t} ; m\right\rangle$ to the ground state $\left|n, S_{t} ; S_{t}\right\rangle$ during the transport process. Moreover, this magnetic field also leads to the symmetry-breaking with respect to the bias reversal in the AP configuration, but the current remains symmetric in the $\mathrm{P}$ configuration. The TMR is illustrated in Fig. 5(a) for $V_{g}=2.3 \mathrm{mV}$, where the transport is mainly through the ground state $|2,2 ; 2\rangle$ in the cotunneling region. It is found that the spin-relaxation induced TMRvariation is negligibly small in the negative bias voltage, while the spin-relaxation can lead to a great change of TMR varying from positive to negative values in the positive bias voltage. Since the currents $I_{P}$ for both positive and negative bias voltages are suppressed equally by the spin-relaxation in the $\mathrm{P}$ configuration, the above asymmetric behavior of TMR is determined by the transport in the AP configuration only. For this case, there exists a competition between the spin-flip process $\frac{19}{19}$ (induced by the inelastic cotunneling) and the intrinsic spinrelaxation. At the negative bias voltage, the above two mechanisms make the SMM be trapped in the ground state $|2,2 ; 2\rangle$ and the current is constant. However, at the positive bias voltage, the spin-flip process makes the SMM tend to the state $|2,2 ;-2\rangle$, but the spin-relaxation drives it to the state $|2,2 ; 2\rangle$. In consequence, the effect of relaxation can compensate the decrease of $I_{A P}$ induced by the spin-flip. Moreover, the TMR for $V_{g}=1$ $\mathrm{mV}$ [Fig. 5(b)] and $V_{g}=-0.3 \mathrm{mV}$ [Fig. 5(c)] exhibits a similar behavior in the cotunneling region.

In order to understand the TMR behavior in the sequential region at $V_{g}=2.3 \mathrm{mV}$ [see Fig. 5(a)], $I_{P}$ and $I_{A P}$ are plotted in Fig. $5(\mathrm{~d})$ for the relaxation strength $\gamma=0$ and $\gamma=10^{3}$. At the negative bias voltage, both the spin-flip process $11,13,14,19,27$ and the intrinsic spinrelaxation can increase the population probabilities of large positive-eigenvalue states of $S_{t}^{z}$ so that the relaxation induced change of $I_{A P}$ is very small. However, at the positive bias voltage, the current $I_{A P}$ is increased much by the relaxation. On the other hand, for both positive and negative bias voltages in the $\mathrm{P}$ configuration, the spin-relaxation results in the enhancement of sequential transition $|2,2 ; 2\rangle \Leftrightarrow|1,5 / 2 ; 5 / 2\rangle$ for the spindown electrons, which are minority in FM electrodes, and thus $I_{P}$ decreases. As a result, the corresponding TMR can vary from positive to negative values along with the increase of the relaxation strength at a fixed bias volt-

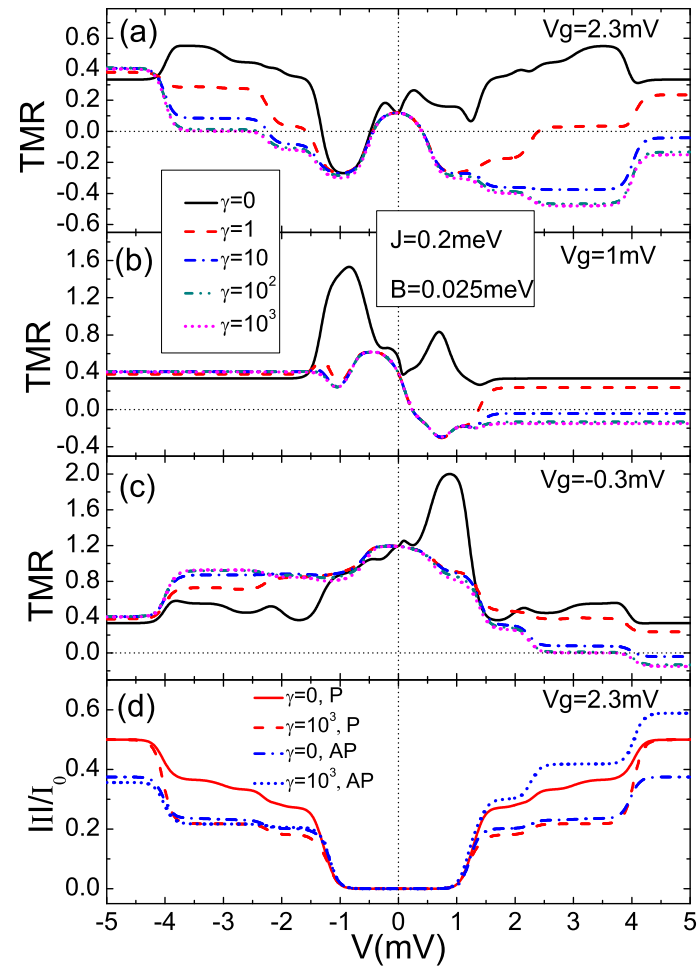

FIG. 5: (Color online) TMR as a function of bias voltage for different gate voltages (a) $V_{g}=2.3 \mathrm{meV}$ (b) $1 \mathrm{meV}$ (c) 0.3 meV. (d) Plots of absolute currents for different relaxation strengths with a magnetic field along the easy-axis of SMM.

age. In particular, a large negative TMR is generated by the fast spin-relaxation at the positive bias voltage. Fig. 5(c) shows the TMR as a function of the bias voltage for $V_{g}=-0.3 \mathrm{mV}$, where the ground state of SMM is $|0,2 ; 2\rangle$. Different from the $V_{g}=2.3 \mathrm{mV}$ case, the TMR is enhanced by the spin-relaxation at the negative bias voltage while it is suppressed even to zero at the positive bias voltage. This is because that the intrinsic spin-relaxation leads to the increase of $I_{P}$ by the enhancement of majority-electron transport through the sequential transition $|0,2 ; 2\rangle \Leftrightarrow|1,5 / 2 ; 5 / 2\rangle$ in the $\mathrm{P}$ configuration. In addition, when the bias voltage is high enough such that all transport channels are open, the $I_{A P}$ is slightly reduced by the spin-relaxation at the negative bias, but it can be greatly enhanced at the positive bias. Therefore, the TMR is slightly above the characteristic value of the sequential region in the negative bias and becomes negative in the positive bias seen from the Figs. 5(a)-5(c).

\section{CONCLUSION}

In summary, the impact of the intrinsic spin-relaxation on electron transport through a SMM with FM leads is investigated by the explicit calculation of spin-relaxation dependence of transport quantities such as current, dif- 
ferential conductance, magnetization and TMR in both the sequential and cotunneling regions. The differential conductance peaks can be completely suppressed by the spin-relaxation for the transition from initial excitedstates in the $\mathrm{P}$ configuration. The magnetization varies from a large negative value to zero with the increase of spin-relaxation strength in the AP configuration, and the current $I_{A P}$ is enhanced. Therefore, the TMR is completely suppressed in the sequential region and can vary from a large positive to slight negative value in the cotunneling region. When an external magnetic field is applied along the easy-axis of SMM, the TMR is asymmetric with respect to the bias reversal. For the cotunneling process, the TMR is much more sensitive to the variation of spin-relaxation in positive bias than in negative bias, especially, it can change from positive to negative values in the former case. In the sequential region, a large negative TMR value can be generated by the spin-relaxation in positive bias. Finally, in the high bias limit the TMR at negative bias is slightly larger than the typical sequential TMR value, while at positive bias it becomes negative due to the spin-relaxation. It is expected that the above transport properties can be observed experimentally in FM-SMM-FM spin valves 38 .

\section{ACKNOWLEDGMENT}

This work was supported by National Natural Science Foundation of China (Grant Nos. 11075099, 11275118, 11204203 and 11004124).
* Electronic address: jqliang@sxu.edu.cn

${ }^{1}$ L. Bogani and W. Wernsdorfer, Nature Mater. 7, 179 (2008).

2 F. Elste and C. Timm, Phys. Rev. B 71, 155403 (2005).

3 G.-H. Kim and T.-S. Kim, Phys. Rev. Lett. 92, 137203 (2004); C. Romeike, M. R. Wegewijs, and H. Schoeller, Phys. Rev. Lett. 96, 196805 (2006); J. Fernández-Rossier and R. Aguado, Phys. Rev. Lett. 98, 106805 (2007); J. Lehmann and D. Loss, Phys. Rev. Lett. 98, 117203 (2007).

${ }^{4}$ H. B. Heersche, Z. de Groot, J. A. Folk, H. S. J. van der Zant, C. Romeike, M. R. Wegewijs, L. Zobbi, D. Barreca, E. Tondello, and A. Cornia, Phys. Rev. Lett. 96, 206801 (2006); M.-H. Jo, J. E. Grose, K. Baheti, M. M. Deshmukh, J. J. Sokol, E. M. Rumberger, D. N. Hendrickson, J. R. Long, H. Park, and D. C. Ralph, Nano Lett. 6, 2014 (2006).

5 M. N. Leuenberger and E. R. Mucciolo, Phys. Rev. Lett. 97, 126601 (2006); G. González and M. N. Leuenberger, Phys. Rev. Lett. 98, 256804 (2007).

${ }^{6}$ C. Romeike, M. R. Wegewijs, W. Hofstetter, and H. Schoeller, Phys. Rev. Lett. 96, 196601 (2006); C. Romeike, M. R. Wegewijs, W. Hofstetter, and H. Schoeller, Phys. Rev. Lett. 97, 206601 (2006); G. Gonzalez, M. N. Leuenberger, and E. R. Mucciolo, Phys. Rev. B 78, 054445 (2008); R.-Q. Wang and D. Y. Xing, Phys. Rev. B 79, 193406 (2009); F. Elste and C. Timm, Phys. Rev. B 81, $024421(2010)$.

7 K.-I. Imura, Y. Utsumi, and T. Martin, Phys. Rev. B 75, 205341 (2007); H.-B. Xue, Y.-H. Nie, Z.-J. Li, and J.-Q. Liang, J. Appl. Phys. 108, 033707 (2010); H.-B. Xue, Y.-H. Nie, Z.-J. Li, and J.-Q. Liang, J. Appl. Phys. 109, 083706 (2011); H.-B. Xue, Y.-H. Nie, Z.-J. Li, and J.-Q. Liang, Phys. Lett. A 375, 716 (2011).

8 T. Jonckheere, K.-I. Imura, and T. Martin, Phys. Rev. B 78, 045316 (2008).

9 Z. Zhang, L. Jiang, R. Wang, B. Wang, and D. Y. Xing, Appl. Phys. Lett. 99, 133110 (2011).

10 H.-Z. Lu, B. Zhou, and S.-Q. Shen, Phys. Rev. B 79, 174419 (2009).

11 M. Misiorny and J. Barnaś, Phys. Rev. B 75, 134425 (2007); Phys. Rev. B 76, 054448 (2007).

12 M. Misiorny and J. Barnaś, Phys. Rev. B 77, 172414 (2008).
13 C. Timm and F. Elste, Phys. Rev. B 73, 235304 (2006).

14 F. Delgado and J. Fernández-Rossier, Phys. Rev. B 82, 134414 (2010).

15 H. Xie, Q. Wang, B. Chang, H. Jiao, and J.-Q. Liang, J. Appl. Phys. 111, 063707 (2012).

16 R.-Q. Wang, L. Sheng, R. Shen, B. Wang, and D.Y. Xing, Phys. Rev. Lett. 105, 057202 (2010); Z. Zhang, L. Jiang, R. Wang, B. Wang, and D. Y. Xing, Appl. Phys. Lett. 97, 242101 (2010).

17 M. Misiorny and J. Barnaś, Europhys. Lett. 89, 18003 (2010).

18 C. Timm and M. Di Ventra, Phys. Rev. B 86, 104427 (2012).

19 M. Misiorny, I. Weymann, and J. Barnaś, Phys. Rev. B 79, 224420 (2009).

20 M. Misiorny, I. Weymann, and J. Barnaś, Phys. Rev. Lett. 106, 126602 (2011); Phys. Rev. B 84, 035445 (2011).

21 M. Misiorny, I. Weymann, and J. Barnaś, J. Appl. Phys. 109, 07C732 (2011).

22 H. Xie, Q. Wang, H. Jiao, and J.-Q. Liang, J. Appl. Phys. 112, 043701 (2012).

${ }^{23}$ F. Elste and C. Timm, Phys. Rev. B 75, 195341 (2007).

24 S. Loth, K. von Bergmann, M. Ternes, A. F. Otte1, C. P. Lutz, and A. J. Heinrich, Nature Physics 6, 340 (2010).

25 C.F. Hirjibehedin, et al., Science 317, 1199 (2007).

26 B. Sothmann and J. König, New J. Phys. 12, 083028 (2010).

27 F. Elste and C. Timm, Phys. Rev. B 73, 235305 (2006).

28 M. Jullière, Phys. Lett. 54A, 225 (1975).

29 M. N. Leuenberger and D. Loss, Nature 410, 789 (2001); B. Zhou, R. Tao, S.-Q. Shen, and J.-Q. Liang, Phys. Rev. A 66, 010301(R) (2002).

30 W. Rudziński and J. Barnaś, Phys. Rev. B 64, 085318 (2001).

31 I. Weymann and J. Barnaś, Phys. Rev. B 73, 205309 (2006).

32 D. Becker and D. Pfannkuche, Phys. Rev. B 77, 205307 (2008).

33 H. Bruus and K. Flensberg, Many-body Quantum Theory in Condensed Matter Physics (Oxford University Press, Oxford, 2004).

34 C. Timm, Phys. Rev. B 77, 195416 (2008). 
35 J. Koch, F. von Oppen, Y. Oreg, and E. Sela, Phys. Rev. B 70, 195107 (2004).

36 J. Koch, F. von Oppen, and A. V. Andreev, Phys. Rev. B 74, 205438 (2006).
37 C. Timm, Phys. Rev. B 83, 115416 (2011).

38 A. Mugarza et al., Phys. Rev. Lett. 107, 177205 (2011). 